\title{
Liver Abscesses Caused by Streptococcus intermedius in an Immunocompromised Patient
}

\author{
Sireesha Reddy ${ }^{1}$, Kalbir Singh ${ }^{2}$, Susan Hughes ${ }^{3}$ \\ 1. University of California, San Francisco - Fresno Department of Family \& Community Medicine 2. \\ Internal Medicine/ Residency Program, San Joaquin General Hospital 3. Hospice and Palliative Medicine, \\ University of California, San Francisco - Fresno Department of Family \& Community Medicine
}

$\square$ Corresponding author: Sireesha Reddy, sireeshareddy@fresno.ucsf.edu Disclosures can be found in Additional Information at the end of the article

\section{Abstract}

Streptococcus intermedius is a Gram-positive bacterium that is part of the normal flora in the oral cavity, as well as in the upper respiratory, female urogenital, and gastrointestinal tracts. It may also be found in human feces and is the dominant species found in subgingival plaque. We present a case of a 50-year-old man who came to the emergency room with abdominal pain for weeks associated with nausea and vomiting, diarrhea, and fever. A computed tomography (CT) scan of the abdomen showed numerous cysts throughout the liver. The patient was started on intravenous (IV) antibiotics and fluid from two of the liver abscesses was obtained for culture.

Categories: Family/General Practice, Infectious Disease

Keywords: streptococcus intermedius, immunocompromised

\section{Introduction}

Streptococcus intermedius is a Gram-positive bacterium that is part of the normal flora in the oral cavity, as well as in the upper respiratory, female urogenital, and gastrointestinal tracts. It may also be found in human feces and is the dominant species found in subgingival plaque.

The "Streptococcus milleri group" (SMG) has been known by a variety of names and, in the past, has been considered a single species that is loosely synonymous with Streptococcus (S.) anginosus. Members of the SMG are now separated into three distinct speciesStreptococcus intermedius, Streptococcus constellatus, and Streptococcus anginosus-with S. constellatus and S. intermedius being more closely related to each other than to S. anginosus. However, because many phenotypic tests for the characterization of these species yield similar results, the identification of isolates can be difficult. S. intermedius tends to be associated with an infection of the head, neck, and respiratory tract. One of the most striking features of species in the SMG is their tendency to cause abscesses. However, both the potential of each of the SMG species to cause abscesses and the clinical features of the cases associated with abscesses have not been addressed in depth with regard to well-identified strains [1].

Reddy et al. This is an open access article distributed under the terms of the Creative Commons Attribution License CC-BY 3.0., which permits unrestricted use, distribution, and reproduction in any medium, provided the original author and source are credited.

Received 11/03/2017 Review ended 01/19/2018 Published 01/24/2018

\begin{abstract}
Streptococcus intermedius is usually found as a solitary isolate associated with deep-seated purulent abscesses, typically found in the brain or liver, central nervous system infections, and infective endocarditis [2-6]. Other articles concluded that undiagnosed diabetes, undiagnosed squamous cell carcinoma, and parenteral drug abusers were common among patients who tested positive for Streptococcus intermedius [2-8].
\end{abstract}




\section{Cureus}

\section{Case Presentation}

A 50-year-old man came to the emergency room with generalized abdominal pain for weeks associated with nausea, vomiting, diarrhea, and fever. Abnormal lab findings included white blood cells (WBCs): 21.0 (4-11 per microliter), total bilirubin: $2.9(0.3-1.2 \mathrm{mg} / \mathrm{dl})$, aspartate aminotransferase (AST): 62 (8-40 U/L), alanine transaminase (ALT): 75 (10-40 U/L), and alkaline phosphatase: 168 (25-100 U/L). The emergency room provider ordered a CT scan of the abdomen. Findings for this patient, as shown in Figure 1, were "numerous, complex, predominantly cystic masses seen throughout the liver; $4 \mathrm{~cm}$ cystic collection interposed between the tip of the right lobe of the liver and hepatic flexure of the colon." At this point, the patient was admitted and symptomatic, and empiric treatments of IV levofloxacin and IV metronidazole were started.

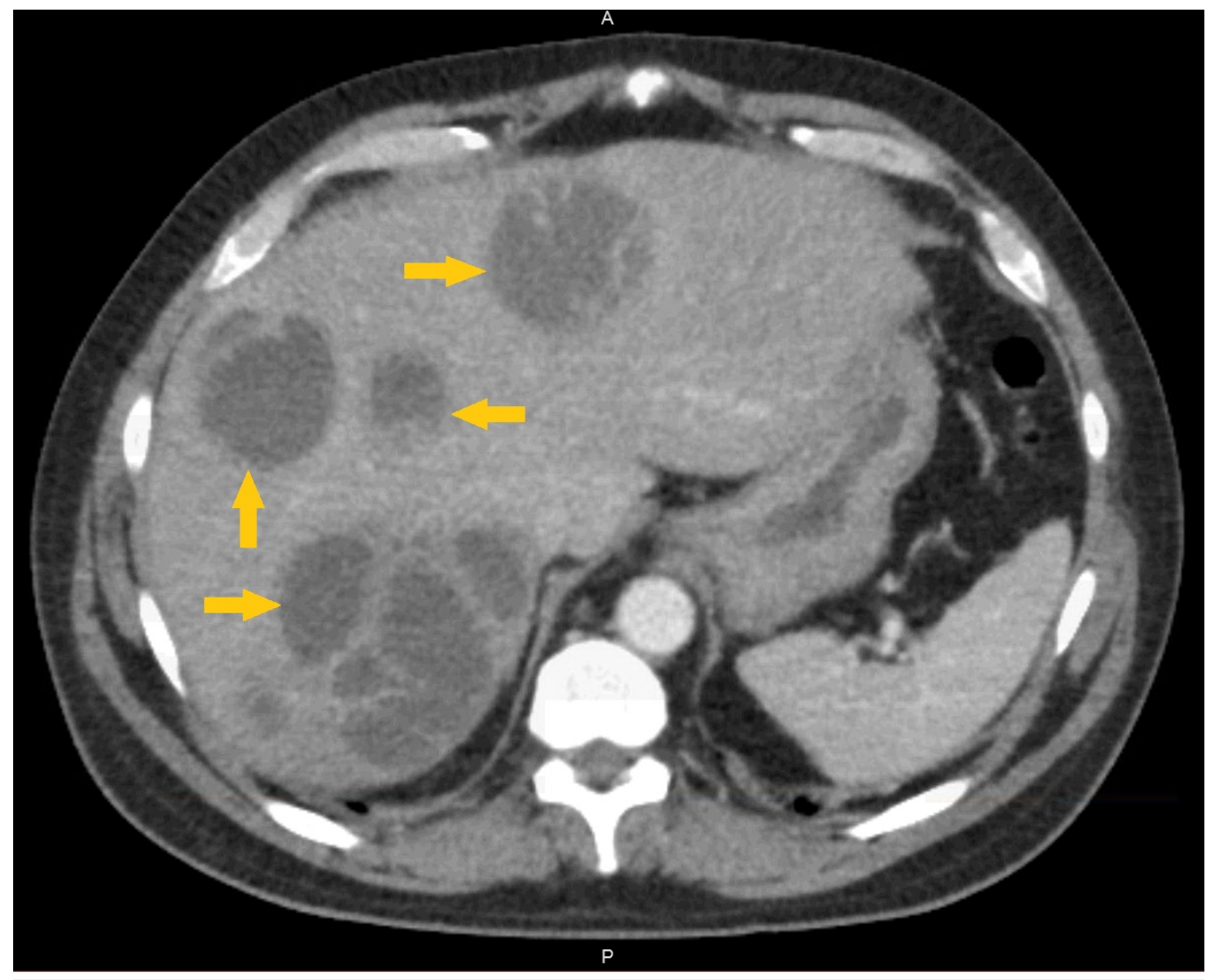

\section{FIGURE 1: Initial CT scan of abdomen with arrows indicating multiple liver abscesses}

CT: computed tomography

Further history of this patient: no travel, no pet exposure, but two teeth extracted one month prior to admission. A diagnosis of Streptococcus intermedius was made from the drainage and culture of the fluid from the liver abscesses, which the lab noted with a failure to thrive for antibiotic susceptibility testing. The blood culture grew Entamoeba histolytica, but it was not detected in the stool.

Our patient was treated initially with IV levofloxacin and metronidazole for two weeks, which was then changed to IV meropenem per the recommendation of the infectious disease specialist. Further abscesses were drained by an interventional radiologist at the University of 


\section{Cureus}

California, San Francisco. The patient was afebrile after starting IV meropenem. Meropenem was given for a total of six weeks. A CT scan of the abdomen approximately nine months after initial admission showed complete resolution of the hepatic abscesses, as shown in Figure 2.

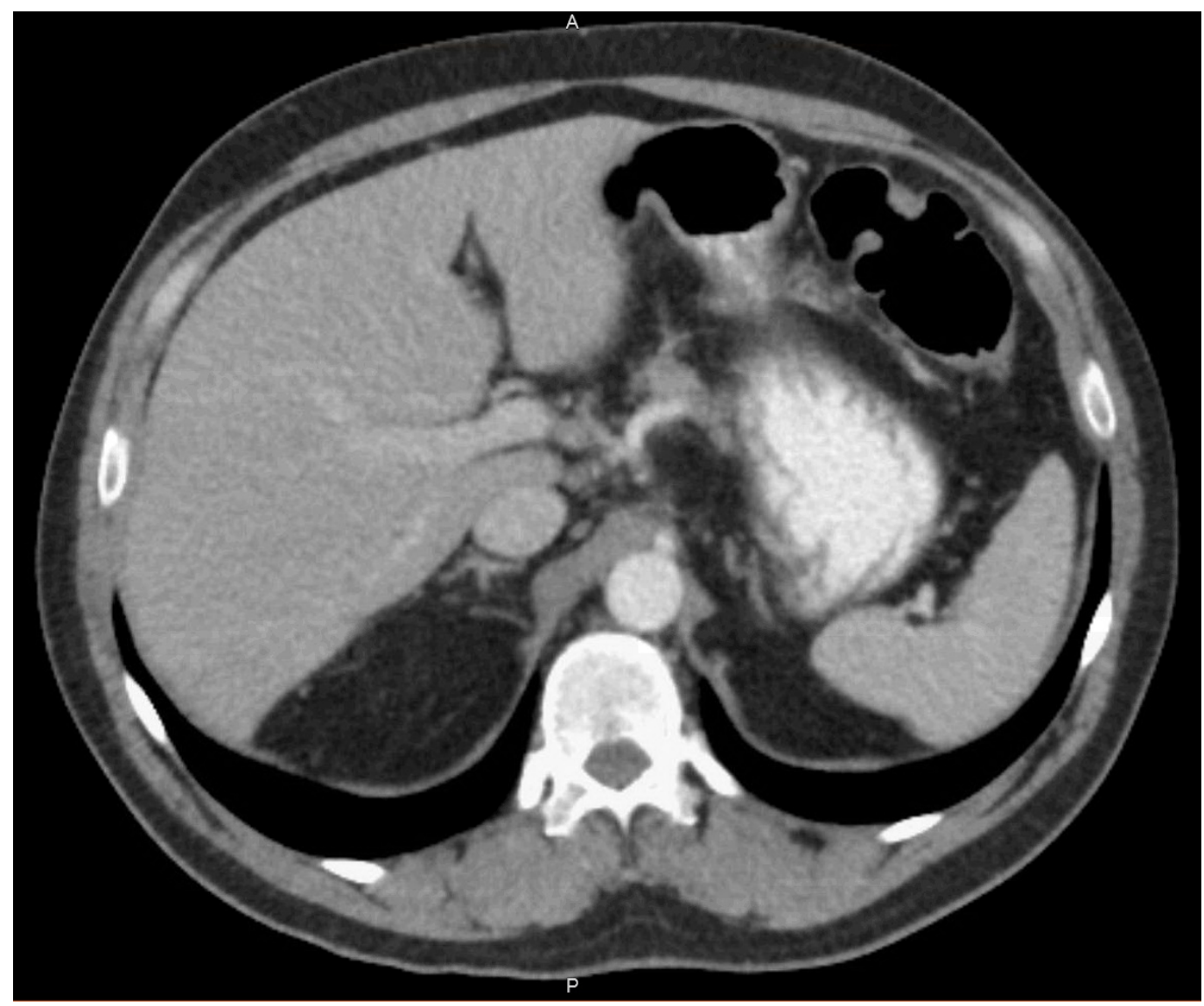

FIGURE 2: CT scan of abdomen after nine months

CT: computed tomography

\section{Discussion}

The unusual findings for this case led us to conduct a further study on other cases of Streptococcus intermedius in our area. We obtained a list of 18 patients testing positive for Streptococcus intermedius from the Community Regional Medical Center, Fresno, California, and Adventist Medical Center, Hanford, California, laboratories from 2010-2015.

We identified patients by a retrospective electronic medical record review. Data collected included verification for Streptococcus intermedius from respective cyst/abscess drainage, abscess location, immune status, co-morbidities, and history of exposures including, but not limited to, dental work, food, or travel.

We defined immunocompromised as any patient with coronary artery disease, diabetes mellitus, cancer, intravenous drug use, human immunodeficiency virus/acquired immunodeficiency syndrome (HIV/AIDS), or hypertension [9].

The risk factors leading to the immunocompromised state of our sample and the development of abscesses were the following: 
- $\quad 56 \%$ had chronic conditions (diabetes mellitus type 2, hypertension, AIDS, or cancer).

- $\quad 33 \%$ had lifestyle choices (alcohol use, tobacco use, raw beef intake, or intravenous drug abuse).

- $11 \%$ had a surgical intervention (dental work or laparoscopic cholecystectomy).

The majority of cases were detected in $2015(n=7)$.

Previous studies have shown this uncommon organism involves mainly diabetic patients and parenteral drug abusers with an intra-abdominal suppurative source in the skin and soft tissues. It is commonly isolated from patients with endocarditis, peritonitis, emphysema, and hepatic and appendiceal abscesses. An aggressive course of antibiotics and control of comorbidities (e.g., diabetes and hypertension) might lead to earlier symptom-free status in patients with dental issues [2-8].

There were several limitations to our study. First, it was retrospective and the documentation of risk factors was done by multiple providers. The small number of cases limited the possibility of defining a relationship between abscess locations and risk factors. An increase in cases in 2015 was likely due to the ability to do full speciation of Streptococcal anginosus. Finally, the duration of risk factors varied as well as the length and state of chronic conditions in individual patients.

\section{Conclusions}

The direct relationship between Streptococcus intermedius as a bacterium with the development of abscesses is not always clear. However, this case report demonstrates a possible association of positive Streptococcus intermedius abscesses developing in patients who are in some form of immunocompromised state.

To understand the etiology of this bacteria, further studies are needed to correlate a relationship between immune status and bacteria.

\section{Additional Information}

\section{Disclosures}

Human subjects: Consent was obtained by all participants in this study. Community Medical Centers Institutional Review Board issued approval 2015075. All documents for the abovereferenced study were reviewed via an expedited review by the Chairman of the Community Medical Centers Institutional Review Board on September 11, 2015. . Conflicts of interest: In compliance with the ICMJE uniform disclosure form, all authors declare the following:

Payment/services info: All authors have declared that no financial support was received from any organization for the submitted work. Financial relationships: All authors have declared that they have no financial relationships at present or within the previous three years with any organizations that might have an interest in the submitted work. Other relationships: All authors have declared that there are no other relationships or activities that could appear to have influenced the submitted work.

\section{References}

1. Clarridge JE III, Attorri S, Musher DM, Hebert J, Dunbar S: Streptococcus intermedius, Streptococcus constellatus, and Streptococcus anginosus (“Streptococcus milleri group”) are of different clinical importance and are not equally associated with abscess. Clin Infect Dis. 


\section{Cureus}

2001, 32:1511-1515. 10.1086/320163

2. Neumayr A, Kubitz R, Bode JG, Bilk B, Häussinger D: Multiple liver abscesses with isolation of Streptococcus intermedius related to a pyogenic dental infection in an immuno-competent patient. Eur J Med Res. 2010, 15:319-322. 10.1186/2047-783X-15-7-319

3. Streptococcus intermedius. (2014). Accessed: 12/12/2015:

http://microbewiki.kenyon.edu/index.php/Streptococcus_intermedius.

4. Melo JC, Raff MJ: Brain abscess due to Streptococcus MG-intermedius (Streptococcus milleri). J Clin Microbiol. 1978, 6:529-532.

5. Poole PM, Wilson G: Occurrence and cultural features of Streptococcus milleri in various body sites. J Clin Pathol. 1979, 8:764-768.

6. Whiley RA, Beighton D, Winstanley TG, Fraser HY, Hardie JM: Streptococcus intermedius, Streptococcus constellatus, and Streptococcus anginosus (the Streptococcus milleri group): association with different body sites and clinical infections. J Clin Microbiol. 1992, 1:243-244.

7. Nayfe R, Ascha MS, Rehmus EH: Esophageal squamous cell carcinoma presenting with Streptococcus intermedius cerebral abscess. Case Rep Pathol. 2017, 2017:5819676. $10.1155 / 2017 / 5819676$

8. Sanchez-Porta A, Torres-Tortosa M, Canueto J, Dominguez MC: Bacteremias due to the Streptococcus milleri group. An analysis of 18 episodes. Rev Clin Esp. 1997, 197:393-397.

9. immunocompromised. (2015). Accessed: 12/12/2015: https://www.merriamwebster.com/dictionary/immunocompromised\#medicalDictionary. 